\title{
Free vibration analysis of a rectangular plate with Kelvin type boundary conditions
}

\author{
R. Kırış1k ${ }^{\mathrm{a}, *}$ and Ş. Yüksel ${ }^{\mathrm{b}}$ \\ ${ }^{\mathrm{a}}$ Turkish Atomic Energy Authority, Eskişehir Yolu 9.km 06530 Lodumlu, Ankara, Turkey \\ ${ }^{\mathrm{b}}$ Department of Mechanical Engineering, Faculty of Engineering and Architecture, Gazi University, 06570 \\ Maltepe, Ankara, Turkey
}

Received 6 August 2006

Revised October 2006

\begin{abstract}
The transverse vibrations of a rectangular plate with the Kelvin type boundary conditions at four corners are investigated. The plate is modeled as being attached to four lumped spring-damper systems at the corners. An analytical procedure is proposed based on the modal analysis. The completely free case of the plate is first studied. The expressions for the eigenfrequencies and eigenfunctions of the plate are obtained by utilizing the separation of variables. Then, the case in which the stiffness and the viscous damping as external forces acting at the corners of the plate is studied. Following the modal analysis procedure, the general solution for the equation of motion of the rectangular plate is derived. Some numerical results are presented.
\end{abstract}

\section{Introduction}

Having many applications in mechanical, civil and aerospace engineerings, the flexure of rectangular plates has been extensively studied in the literature. The earliest works on this subject which covered the classical theory with some restrictions were reviewed in the Leissa's book [8]. Following that, considering a variety of boundary conditions, Leissa [9] analyzed twenty-one cases of rectangular plates which involved the possible combinations of clamped, simply-supported, and free-edge boundary conditions. The author showed the effects of changing edge conditions upon the frequencies and their accuracies, and also the effects of changing Poisson's ratio upon the vibration frequencies. In another study, Leissa et al. [10] solved the problem of simply supported rectangular plate having parabolically varying rotational constraints at the two opposite edges by using both the exact solutions of differential equations and the Ritz method. The numerical results were presented and compared to each other and with other results for certain limiting cases.

By using the method of superposition, Gorman [1] proposed a new theoretical approach to analyze the free vibration of the completely free rectangular plate. The method involves choosing auxiliary plate problems for which accurate solutions are easily obtained, superimposing these solutions, and constraining them such that their combined solution satisfies the boundary conditions of interest. The author provided the eigenvalues with four digit accuracy for a wide range of plate aspect ratios and modal shapes.

Because of its importance in obtaining sufficient engineering accuracy for many practical applications, Laura et al. [6] used polynomial coordinate functions and the Rayleigh-Ritz method to calculate the fundamental frequency coefficient for a rectangular plate with edges elastically restrained against both translation and rotation. They claimed that the approach is simple and straightforward and gives the solution of a rather difficult elastodynamics problem. Using a similar approach, Gutierrez et al. [3] studied the vibrations of a rectangular plate having a thickness which

*Corresponding author. Fax: +90 312287 8761; E-mail: rasit.kirisik@taek.gov.tr. 
varies in bilinear fashion in the $x$-axis. In that study, the authors considered the translational and rotational flexibilities at all edges and presented a simple algorithm which allows one to evaluate the fundamental frequency of vibration.

Laura et al. [7] presented simple analytical methods for calculating the fundamental frequencies of vibration of three types of plates. These are first orthotropic rectangular plates with edges possessing different rotational flexibility parameters, second clamped and simply supported plates of complicated boundary shape, and third isotropic circular plates subjected to a hydrostatic state of stress and elastically restrained against the translation and rotation.

Using a similar approach as in reference [1], Gorman [2] exploited the method of superposition to obtain a solution for the free vibration of thin rectangular plates resting on elastic edge supports of arbitrarily distributed stiffness in which step discontinuities exist. In that study, it was found that the eigenvalues approach their known proper limits as the stiffness approach their limits of zero and infinity. In another study on the mixed boundary conditions, Singhal et al. [12] proposed an analytical procedure based on the method of superposition to obtain the free vibration frequencies and mode shapes of partially clamped cantilevered rectangular plates with and without rigid point supports. They also conducted a number of experimental tests in order to permit comparison between theoretical and experimental results.

Rajalingham et al. [11] presented a method which yields accurate results for the natural frequencies and plate characteristic functions for the clamped rectangular plates. They obtained the optimum separable solutions of the plate vibration equation by reducing it to simultaneous ordinary differential equations. In order to represent various structural types of building floors, the plate may be simultaneously subjected to many different constraints including elastic edge and point supports. Kato et al. [5] adopted the Rayleigh-Ritz solution for the transverse vibration analysis of a thin rectangular plate in order to estimate the vibration characteristic of building floors.

Recently, Zhao et al. [13] succeeded to introduce the discrete singular convolution to the vibration analysis of rectangular plates with non-uniform and combined boundary conditions. They employed twenty one non-trivial cases constructed from all possible boundary condition combinations of simply supported, clamped and transversely supported edges.

Most of the previous studies related to the flexure of rectangular plates with classical theory, a variety of boundary conditions have been taken into consideration. These boundary conditions are generally possible combinations of simple-supported, clamped, and free edges. Additionally, the rectangular plates with rigid point supports, distributed elastic and rigid supports, and elastic edges restrained against both translation and rotation have also been investigated.

In the present study, the transverse vibrations of a rectangular plate with the Kelvin type boundary conditions including stiffness and viscous damping at four corners are investigated. Here, the classical theory is used for the equation of motion of the plate and an analytical procedure is proposed based on the modal analysis.

At first, the continuous model of the free-free plate is studied. By using the separation of variables, the eigenfrequencies and eigenfunctions are derived. After that, the new case is studied by using the Kelvin type boundary conditions at four corners. The spring and viscous damping forces are assumed to act as external forces at the corners of the plate. Then, using the modal analysis procedure, the general solution to the equation of motion of this damped rectangular plate is obtained. Finally, the results of sample numerical calculations are carried out for undamped and damped cases.

\section{Problem statement and formulation}

The plate model considered in this study is shown in Fig. 1. It consists of a transversely vibrating plate with Kelvin type boundary conditions at four corners. The following expression is the fundamental differential equation in the classical theory of vibration of plates [4]

$$
D\left(\frac{\partial^{4} w(x, y, t)}{\partial x^{4}}+2 \frac{\partial^{4} w(x, y, t)}{\partial x^{2} \partial y^{2}}+\frac{\partial^{4} w(x, y, t)}{\partial y^{4}}\right)=-\rho \frac{\partial^{2} w(x, y, t)}{\partial t^{2}}
$$

where $w(x, y, t)$ denotes the vertical displacement of a typical element at a position $(x, y), \rho$ denotes the mass per unit area of the plate, and $t$ denotes the time. Additionally, $D$ represents the flexural rigidity of the plate, defined as

$$
D=\frac{E h^{3}}{12\left(1-\nu^{2}\right)}
$$




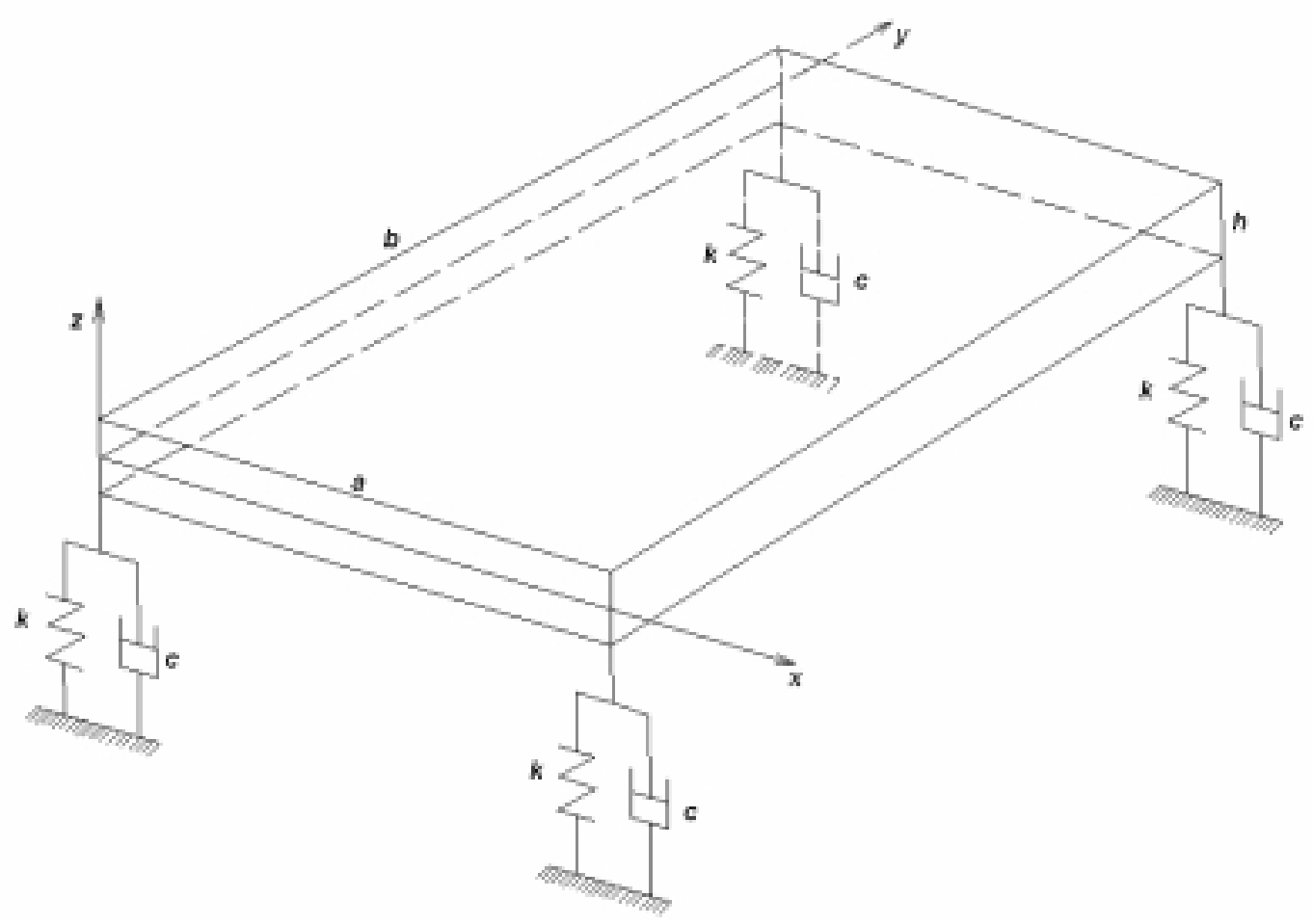

Fig. 1. Rectangular plate with Kelvin type boundary conditions at four corners.

where $E$ shows the modulus of elasticity, $h$ shows the thickness of the plate, and $v$ shows Poisson's ratio.

Here, the equation of motion of the rectangular plate with Kelvin type boundary conditions is solved first for the free edges case.

\subsection{Completely free plate}

The equation of motion expressed by Eq. (1) is to be solved with the following four boundary conditions, considering the symmetry of the boundary conditions;

$$
\begin{aligned}
& M_{y(y=0)}=0, \quad M_{y(y=b)}=0 \\
& V_{y(y=0)}=0, \quad V_{y(y=b)}=0
\end{aligned}
$$

where $M_{y}$ is the bending moment, and $V_{y}$ is the transverse shear force in $y$-direction.

For the other edges of the plate, a sliding edge or a slip shear support is supposed in which the slope is zero and no shear force is allowed [4].

It is assumed that the displacement $w(x, y, t)$ can be written as the product of two functions. Namely, a function $W$ which depends on the spatial coordinates $x, y$ and a function $f$ which is a time-dependent harmonic function of frequency $\omega$. Thus,

$$
w(x, y, t)=W(x, y) f(t)
$$

Substitution of this separated form into the equation of motion given by Eq. (1) yields the following two new differential equations; one of which is an ordinary differential equation and the other is a partial differential equation

$$
-\frac{d^{2} f(t)}{d t^{2}}=\omega^{2} f(t)
$$




$$
\frac{D}{\rho}\left(\frac{\partial^{4} W(x, y)}{\partial x^{4}}+2 \frac{\partial^{4} W(x, y)}{\partial x^{2} \partial y^{2}}+\frac{\partial^{4} W(x, y)}{\partial y^{4}}\right)=\omega^{2} W(x, y)
$$

The solution of the first differential equation given by Eq. (6) is well known and determined by the initial conditions. The second differential equation given by Eq. (7) determines the mode forms of the plate. Now, one can consider the mode forms of the following type:

$$
\begin{aligned}
W_{m}(x, y)= & {\left[C_{1} \sin \left(\alpha_{m} y\right)+C_{2} \cos \left(\alpha_{m} y\right)+C_{3} \sinh \left(\gamma_{m} y\right)+C_{4} \cosh \left(\gamma_{m} y\right)\right] \cos \left(\frac{m \pi x}{a}\right) } \\
& m=1,2,3 \ldots
\end{aligned}
$$

as a multiplication of mode shape of a free-free beam along $y$ and cosine mode shape along $x$ since the assumption of the free edges and the slip-shear support. Where the notations

$$
\begin{aligned}
& \alpha_{m}^{2}=\omega \sqrt{\frac{\rho}{D}}-\left(m \frac{\pi}{a}\right)^{2} \\
& \gamma_{m}^{2}=\omega \sqrt{\frac{\rho}{D}}+\left(m \frac{\pi}{a}\right)^{2}
\end{aligned}
$$

are introduced for simplicity. In Eq. (8), $C_{1}, C_{2}, C_{3}$ and $C_{4}$ are the integration constants to be determined by the boundary conditions.

The boundary conditions given by Eqs (3) and (4) have the general form of

$$
\begin{aligned}
& M_{y}=-D\left(\frac{\partial^{2} W(x, y)}{\partial y^{2}}+\nu \frac{\partial^{2} W(x, y)}{\partial x^{2}}\right) \\
& V_{y}=-D\left(\frac{\partial^{3} W(x, y)}{\partial y^{3}}+(2-\nu) \frac{\partial^{2} W(x, y)}{\partial x^{2} \partial y}\right)
\end{aligned}
$$

The application of boundary conditions given by Eqs (3) and (4) in connection with the expressions (10) and (11) results in

$$
\begin{aligned}
& \left(-\alpha_{m}^{2}-r_{m}^{2} \nu\right) C_{2}+\left(\gamma_{m}^{2}-r_{m}^{2} \nu\right) C_{4}=0 \\
& {\left[-\alpha_{m}^{3}-(2-\nu) r_{m}^{2} \alpha_{m}\right] C_{1}+\left[\gamma_{m}^{3}-(2-\nu) r_{m}^{2} \gamma_{m}\right] C_{3}=0} \\
& \left(-\alpha_{m}^{2}-r_{m}^{2} \nu\right) \sin \left(\alpha_{m} b\right) C_{1}+\left(-\alpha_{m}^{2}-r_{m}^{2} \nu\right) \cos \left(\alpha_{m} b\right) C_{2} \\
& \quad+\left(\gamma_{m}^{2}-r_{m}^{2} \nu\right) \sinh \left(\gamma_{m} b\right) C_{3}+\left(\gamma_{m}^{2}-r_{m}^{2} \nu\right) \cosh \left(\gamma_{m} b\right) C_{4}=0 \\
& {\left[-\alpha_{m}^{3}-(2-\nu) r_{m}^{2} \alpha_{m}\right] \cos \left(\alpha_{m} b\right) C_{1}+\left[\alpha_{m}^{3}-(2-\nu) r_{m}^{2} \alpha_{m}\right] \sin \left(\alpha_{m} b\right) C_{2}} \\
& \quad+\left[\gamma_{m}^{3}-(2-\nu) r_{m}^{2} \gamma_{m}\right] \cosh \left(\gamma_{m} b\right) C_{3}+\left[\gamma_{m}^{3}-(2-\nu) r_{m}^{2} \gamma_{m}\right] \sinh \left(\gamma_{m} b\right) C_{4}=0
\end{aligned}
$$

where

$$
r_{m}=m \frac{\pi}{a} ; \quad m=1,2,3, \ldots
$$

The four linear algebraic equations given by Eqs (12) through (15) determine the values of $C_{j}(j=1,2,3, \ldots)$ for each positive integer $m$. The values of the constants are not all zero if, and only if, the determinant of coefficient matrix is zero. This condition yields the characteristic equation which can be solved numerically for the values of eigenfrequencies $\omega_{m n}(m=1,2,3, \ldots)$ for each positive integer $n$. For each values of $m(m=1,2,3, \ldots)$ there are infinitely many eigenfrequencies. Therefore $\omega_{m n}$ is used instead of $\omega$ to represent these eigenfrequencies.

The individual modes of vibration can be calculated with these values. Solving the equations given by Eqs (12)(15) gives any three of the individual coefficients. The remaining coefficient becomes the arbitrary magnitude of the eigenfunction. Now, for each values of $m$ and $n$, the substitution of these values into expression (8) yields the eigenfunctions or natural mode shapes as 


$$
\begin{aligned}
W_{m n}(x, y)= & B_{m n}\left(B_{1 m n} \sin \left(\alpha_{m n} y\right)+B_{2 m n} \cos \left(\alpha_{m n} y\right)\right. \\
& \left.B_{3 m n} \sinh \left(\gamma_{m n} y\right)+\cosh \left(\gamma_{m n} y\right)\right) \cos \frac{m \pi x}{a} ; \quad m, n=1,2,3 \ldots
\end{aligned}
$$

where the notations

$$
\begin{aligned}
& B_{1 m n}=\frac{\gamma_{m n}^{3}-(2-\nu) r_{m}^{2} \gamma_{m n}}{\alpha_{m n}^{3}-(2-\nu) r_{m}^{2} \alpha_{m n}} \times \frac{\cosh \left(\gamma_{m n} b\right)-\cos \left(\alpha_{m n} b\right)}{\frac{\left(\alpha_{m n}^{2}+r_{m}^{2} \nu\right)\left(\gamma_{m n}^{3}-(2-\nu) r_{m}^{2} \gamma_{m n}\right)}{\left(\gamma_{m n}^{2}-r_{m}^{2} \nu\right)\left(\alpha_{m n}^{3}-(2-\nu) r_{m}^{2} \alpha_{m n}\right)} \sin \left(\alpha_{m n} b\right)-\sinh \left(\gamma_{m n} b\right)} \\
& B_{2 m n}=\frac{\gamma_{m n}^{2}-r_{m}^{2} \nu}{\alpha_{m n}^{2}+r_{m}^{2} \nu} \\
& B_{3 m n}=\frac{\cosh \left(\gamma_{m n} b\right)-\cos \left(\alpha_{m n} b\right)}{\frac{\left(\alpha_{m n}^{2}+r_{m}^{2} \nu\right)\left(\gamma_{m n}^{3}-(2-\nu) r_{m}^{2} \gamma_{m n}\right)}{\left(\gamma_{m n}^{2}-r_{m}^{2} \nu\right)\left(\alpha_{m n}^{3}-(2-\nu) r_{m}^{2} \alpha_{m n}\right)} \sin \left(\alpha_{m n} b\right)-\sinh \left(\gamma_{m n} b\right)}
\end{aligned}
$$

are introduced for simplicity. Here, $B_{m n}$ is the arbitrary magnitude of the eigenfunction to be determined by the initial conditions.

Because of the linearity and homogeneity of the equation of motion given by Eq. (1), the sum of any number of natural modes is a possible free vibration. Thus, the general solution of the equation can be written in the form of

$$
w(x, y, t)=\sum_{m=1}^{\infty} \sum_{n=1}^{\infty} A_{m n} \sin \left(\omega_{m n} t+\Phi_{m n}\right) W_{m n}(x, y)
$$

where $A_{m n}$ and $\Phi_{m n}$ are the integration constants coming from the solution of the ordinary differential equation given by Eq. (6) and can be determined by the initial conditions.

\subsection{Supported plate}

In this part of the study, the effect of the springs and dampers will be included in the rectangular plate model. Assuming that external spring and viscous damping forces act at the four corners of the plate, the equation of motion becomes [4]

$$
\begin{aligned}
& D \nabla^{4} w(x, y, t)+\delta(x) \delta(y)[k w(x, y, t)+c \dot{w}(x, y, t)] \\
& \quad+\delta(x-a) \delta(y)[k w(x, y, t)+c \dot{w}(x, y, t)]+\delta(x) \delta(y-b)[k w(x, y, t)+c \dot{w}(x, y, t)] \\
& \quad+\delta(x-a) \delta(y-a)[k w(x, y, t)+c \dot{w}(x, y, t)]=-\rho \frac{\partial^{2} w(x, y, t)}{\partial^{2} t}
\end{aligned}
$$

where the biharmonic operator

$$
\nabla^{4}=\frac{\partial^{4}}{\partial x^{4}}+2 \frac{\partial^{4}}{\partial x^{2} \partial y^{2}}+\frac{\partial^{4}}{\partial y^{4}}
$$

and the Dirac's delta function

$$
\begin{aligned}
& \delta(x-a)=0 ; x \neq a \\
& \int_{0}^{\infty} \delta(x-a) d x=1
\end{aligned}
$$

are utilized, and $k$ and $c$ shows the coefficients of spring and viscous damping respectively.

Following the modal analysis procedure, one can assume that the solution of the differential equation is of the form for each eigenfunction of completely free plate [4],

$$
w_{m n}(x, y, t)=W_{m n}(x, y) f_{m n}(t)
$$

Substituting this form into Eq. (22) gives 


$$
\begin{aligned}
& D f_{m n} \nabla^{4} W_{m n}(x, y)+\delta(x) \delta(y)\left[k f_{m n}(t) W_{m n}(x, y)+c W_{m n}(x, y) \dot{f}_{m n}(t)\right] \\
& \quad+\delta(x-a) \delta(y)\left[k f_{m n}(t) W_{m n}(x, y)+c W_{m n}(x, y) \dot{f}_{m n}(t)\right] \\
& \quad+\delta(x) \delta(y-b)\left[k f_{m n}(t) W_{m n}(x, y)+c W_{m n}(x, y) \dot{f}_{m n}(t)\right] \\
& \quad+\delta(x-a) \delta(y-a)\left[k f_{m n}(t) W_{m n}(x, y)+c W_{m n}(x, y) \dot{f}_{m n}(t)\right]=-\rho W_{m n}(x, y) \ddot{f}_{m n}(t) \\
& \quad m, n=1,2,3 \ldots
\end{aligned}
$$

If this equation is integrated over the plate, then

$$
\begin{aligned}
& \int_{0}^{a} \int_{0}^{b} D f_{m n} \nabla^{4} W_{m n}(x, y) d x d y+\int_{0}^{a} \int_{0}^{b}\left\{\delta(x) \delta(y)\left[k f_{m n}(t) W_{m n}(x, y)+c W_{m n}(x, y) \dot{f}_{m n}(t)\right]\right. \\
& \quad+\delta(x-a) \delta(y)\left[k f_{m n}(t) W_{m n}(x, y)+c W_{m n}(x, y) \dot{f}_{m n}(t)\right] \\
& \quad+\delta(x) \delta(y-b)\left[k f_{m n}(t) W_{m n}(x, y)+c W_{m n}(x, y) \dot{f}_{m n}(t)\right] \\
& \left.\quad+\delta(x-a) \delta(y-a)\left[k f_{m n}(t) W_{m n}(x, y)+c W_{m n}(x, y) \dot{f}_{m n}(t)\right]\right\} d x d y \\
& \quad=\int_{0}^{a} \int_{0}^{b}-\rho W_{m n}(x, y) \ddot{f}_{m n}(t) d x d y \\
& m, n=1,2,3 \ldots
\end{aligned}
$$

is obtained. Rearranging the terms of the above equation yields

$$
\rho \bar{P}_{3 m n} \ddot{f}_{m n}(t)+c \bar{P}_{2 m n} \dot{f}_{m n}(t)+\bar{P}_{1 m n} f_{m n}(t)=0 ; \quad m, n=1,2,3, \ldots
$$

where the notations

$$
\begin{aligned}
\bar{P}_{1 m n}= & \int_{0}^{a} \int_{0}^{b} D \nabla^{4} W_{m n}(x, y) d x d y+\int_{0}^{a} \int_{0}^{b}\left\{\delta(x) \delta(y)\left[k W_{m n}(x, y)\right]+\delta(x-a) \delta(y)\left[k W_{m n}(x, y)\right]\right. \\
& \left.+\delta(x) \delta(y-b)\left[k W_{m n}(x, y)\right]+\delta(x-a) \delta(y-a)\left[k W_{m n}(x, y)\right]\right\} d x d y \\
\bar{P}_{2 m n}= & \int_{0}^{a} \int_{0}^{b}\left\{\delta(x) \delta(y)\left[c W_{m n}(x, y)\right]+\delta(x-a) \delta(y)\left[c W_{m n}(x, y)\right]\right. \\
& \left.+\delta(x) \delta(y-b)\left[c W_{m n}(x, y)\right]+\delta(x-a) \delta(y-a)\left[c W_{m n}(x, y)\right]\right\} d x d y \\
\bar{P}_{3 m n}= & \int_{0}^{a} \int_{0}^{b} W_{m n}(x, y) d x d y
\end{aligned}
$$

are introduced for simplicity. Using the eigenfunction expression (17), and utilizing the biharmonic operator and the Dirac's delta function, after lengthy calculations and some manipulations, expression (28) can be rearranged by defining

$$
\begin{aligned}
& P_{1 m n}=D\left\{\begin{array}{c}
\left(\frac{r_{m}^{3}}{\alpha_{m n}}+2 r_{m} \alpha_{m n}+\frac{\alpha_{m n}^{3}}{r_{m}}\right)\left(B_{1 m n}\left(1-\cos \left(\alpha_{m n} b\right)\right)+B_{2 m n} \sin \left(\alpha_{m n} b\right)\right) \\
+\left(\frac{r_{m}^{3}}{\gamma_{m n}}-2 r_{m} \gamma_{m n}+\frac{\gamma_{m n}^{3}}{r_{m}}\right)\left(B_{3 m n}\left(\cosh \left(\gamma_{m n} b\right)-1\right)+\sinh \left(\gamma_{m n} b\right)\right)
\end{array}\right\} \\
& +\frac{k}{r_{m}}\left\{\begin{array}{l}
\frac{-B_{1 m n}}{\alpha_{m n}}\left(1+\cos \left(\alpha_{m n} b\right)\right)+\frac{B_{2 m n}}{\alpha_{m n}} \sin \left(\alpha_{m n} b\right)+ \\
\frac{B_{3 m n}}{\gamma_{m n}}\left(\cosh \left(\gamma_{m n} b\right)+1\right)+\frac{1}{\gamma_{m n}} \sinh \left(\gamma_{m n} b\right)
\end{array}\right\} \\
& P_{2 m n}=\frac{1}{r_{m}}\left\{\begin{array}{l}
\frac{-B_{1 m n}}{\alpha_{m n}}\left(1+\cos \left(\alpha_{m n} b\right)\right)+\frac{B_{2 m n}}{\alpha_{m n}} \sin \left(\alpha_{m n} b\right)+ \\
\frac{B_{3 m n}}{\gamma_{m n}}\left(\cosh \left(\gamma_{m n} b\right)+1\right)+\frac{1}{\gamma_{m n}} \sinh \left(\gamma_{m n} b\right)
\end{array}\right\}
\end{aligned}
$$




$$
P_{3 m n}=\frac{1}{r_{m}}\left\{\begin{array}{l}
\frac{B_{1 m n}}{\alpha_{m n}}\left(1-\cos \left(\alpha_{m n} b\right)\right)+\frac{B_{2 m n}}{\alpha_{m n}} \sin \left(\alpha_{m n} b\right)+ \\
\frac{B_{3 m n}}{\gamma_{m n}}\left(\cosh \left(\gamma_{m n} b\right)-1\right)+\frac{1}{\gamma_{m n}} \sinh \left(\gamma_{m n} b\right)
\end{array}\right\}
$$

then Eq. (28) can be written in the form

$$
\ddot{f}_{m n}(t)+\frac{c P_{2 m n}}{\rho P_{3 m n}} \dot{f}_{m n}(t)+\frac{P_{1 m n}}{\rho P_{3 m n}} f_{m n}(t)=0 ; \quad m, n=1,2,3, \ldots
$$

which is similar to the well-known equation of motion of a damped single degree of freedom system expressed as

$$
\ddot{x}(t)+2 \zeta_{n} \omega_{n} \dot{x}(t)+\omega_{n}^{2} x(t)=0
$$

where $x(t)$ is the displacement and $\mathrm{m}$ is the mass, $\omega_{m n}$ is the undamped natural frequency, and $\zeta_{n}$ is the damping ratio defined as

$$
\zeta_{n}=\frac{c}{2 \mathrm{~m} \omega_{n}}
$$

One can write the following equalities from Eqs (35) and (36) by analogy

$$
\begin{aligned}
& \omega_{m n}^{2}=\frac{P_{1 m n}}{\rho P_{3 m n}} ; m, n=1,2,3, \ldots \ldots \\
& 2 \xi_{m n} \omega_{m n}=\frac{c P_{2 m n}}{\rho P_{3 m n}} ; \quad m, n=1,2,3, \ldots
\end{aligned}
$$

The last two expressions give

$$
\xi_{m n}=\frac{1}{2 \sqrt{\rho}} \frac{c P_{2 m n}}{\sqrt{P_{1 m n} P_{3 m n}}} ; \quad m, n=1,2,3, \ldots
$$

As a result of this analogy, Eqs (38) and (40) give the natural frequencies and damping ratios for the positive integers $m$ and $n$.

Moreover, the solution to Eq. (35) for an underdamped mode can be written as

$$
f_{m n}(t)=A_{m n} e^{-\xi_{m n} \omega_{m n} t} \sin \left(\omega_{d m n} t+\Phi_{m n}\right) ; \quad m, n=1,2,3 \ldots
$$

where the notations

$$
\begin{aligned}
A_{m n} & =\sqrt{\frac{\left(\dot{w}(x, y, 0)+\xi_{m n} \omega_{m n} w(x, y, 0)\right)^{2}+\left(w(x, y, 0) \omega_{d m n}\right)^{2}}{\omega_{d m n}^{2}}} \\
\Phi_{m n} & =\tan ^{-1}\left[\frac{w(x, y, 0) \omega_{d m n}}{\dot{w}(x, y, 0)+\xi_{m n} \omega_{m n} w(x, y, 0)}\right] \\
\omega_{d m n} & =\omega_{m n} \sqrt{1-\xi_{m n}^{2}}
\end{aligned}
$$

are introduced. Here, $A_{m n}$ and $\Phi_{m n}$ are the constants of integration and depend on the initial conditions. Furthermore, $\omega_{d m n}$ denotes the damped natural frequencies.

Finally, combining the solutions (17) and (41) in connection with the assumption (25), and forming the summation over all modes, the total solution of the differential Eq. (26) can be written as

$$
\begin{aligned}
w(x, y, t)= & \sum_{m=1}^{\infty} \sum_{n=1}^{\infty} A_{m n}\left[B_{1 m n} \sin \left(\alpha_{m n} y\right)+B_{2 m n} \cos \left(\alpha_{m n} y\right.\right. \\
& \left.+B_{3 m n} \sinh \left(\gamma_{m n} y\right)+\cosh \left(\gamma_{m n} y\right)\right] \cos (r x) e^{-\xi_{m n} \omega_{m n} t} \sin \left(\omega_{d m n} t+\Phi_{m n}\right)
\end{aligned}
$$

where the integration constants $A_{m n}$ and $\Phi_{m n}$ are to be determined by utilizing the initial conditions. 
Table 1

Dimensionless frequency parameters $\lambda_{m n}$ for square plate and different Poisson's ratios

\begin{tabular}{|c|c|c|c|c|c|c|c|c|}
\hline \multirow{2}{*}{$\begin{array}{c}\text { Mode } \\
\mathrm{m}\end{array}$} & \multicolumn{2}{|c|}{$\nu=0.225$} & \multicolumn{2}{|c|}{$\nu=0.343$} & \multicolumn{2}{|c|}{$\nu=0.360$} & \multicolumn{2}{|c|}{$\nu=0.360$} \\
\hline & & Leissa & & Leissa & & Leissa & & Leissa \\
\hline 1 & 13.054 & 14.14 & 12.585 & 13.10 & 12.499 & 12.94 & 12.337 & 12.64 \\
\hline 2 & 22.373 & 20.49 & 22.378 & 19.306 & 22.226 & 19.129 & 21.937 & 18.707 \\
\hline 3 & 29.461 & 23.97 & 28.402 & 24.64 & 28.209 & 24.73 & 27.842 & 24.8 \\
\hline 4 & 61.673 & 66.402 & 59.456 & 63.16 & 59.052 & 62.664 & 58.284 & 61.329 \\
\hline 5 & 71.946 & 71.83 & 69.36 & 68.50 & 68.888 & 67.993 & 67.992 & 66.82 \\
\hline 6 & 75.873 & 77.881 & 73.146 & 77.73 & 72.649 & 77.683 & 71.703 & 77.162 \\
\hline
\end{tabular}

Table 2

Dimensionless damping ratios and damped frequency parameters $\lambda_{d m n}$ for different Poisson's ratios

\begin{tabular}{|c|c|c|c|c|c|c|c|c|}
\hline \multirow{2}{*}{$\begin{array}{c}\text { Mode } \\
\mathrm{m}\end{array}$} & \multicolumn{2}{|c|}{$\nu=0.225$} & \multicolumn{2}{|c|}{$\nu=0.343$} & \multicolumn{2}{|c|}{$\nu=0.360$} & \multicolumn{2}{|c|}{$\nu=0.360$} \\
\hline & $\xi$ & $\lambda_{d}$ & $\xi$ & $\lambda_{d}$ & $\xi$ & $\lambda_{d}$ & $\xi$ & $\lambda_{d}$ \\
\hline 1 & 0.531 & 11.064 & 0.678 & 9.253 & 0.701 & 8.913 & 0.745 & 8.232 \\
\hline 2 & 0.267 & 21.562 & 0.266 & 21.57 & 0.266 & 21.424 & 0.266 & 21.148 \\
\hline 3 & 0.265 & 28.412 & 0.265 & 28.39 & 0.265 & 27.204 & 0.265 & 26.85 \\
\hline 4 & 0.264 & 59.486 & 0.264 & 57.349 & 0.264 & 56.96 & 0.264 & 56.219 \\
\hline 5 & 0.263 & 69.42 & 0.262 & 69.933 & 0.262 & 66.479 & 0.262 & 65.616 \\
\hline 6 & 0.26 & 73.269 & 0.259 & 70.657 & 0.258 & 70.179 & 0.258 & 69.272 \\
\hline
\end{tabular}

\section{Numerical results}

In this section, the numerical results of the analytical solutions for the eigenfrequencies and the damping ratios are presented. A realistic model is studied by using a plate for which the density is $\rho=2710 \mathrm{~kg} / \mathrm{m}^{3}$, the modulus of elasticity is $E=71 \times 10^{9} \mathrm{~N} / \mathrm{m}^{2}$, and various Poisson's ratios that can vary between 0 and 0.5 for isotropic materials. A rectangular plate with dimension $a=9 \mathrm{~m}$, and different aspect ratios $a / b=0.4,1.0,2.5$, and a thickness of $h=$ $9.0 \times 10^{-3} \mathrm{~m}$ is assumed. At the corners, the spring coefficient $k=15 \times 10^{5} \mathrm{~N} / \mathrm{m}$ and the damping coefficient $c=$ $32 \times 10^{2} \mathrm{Ns} / \mathrm{m}$ are used for the Kelvin type boundary conditions.

Table 1 presents the dimensionless frequency parameters for the free edges case for various Poisson's ratios and square plate, defined by

$$
\lambda_{m n}=\omega_{m n} a^{2} \sqrt{\frac{\rho}{D}}
$$

where the eigenfrequencies $\omega_{m n}$ are solved from the characteristic equation which is obtained from the determinant of the coefficient matrix of the algebraic Eqs (12)-(15) for positive integer values of $m$ and $n$. The results are fairly good agreement with the values given in the literature.

In Table 1, the dimensionless frequency parameters are slightly different from the ones calculated by Leissa since the admissible functions that are used in both studies are different. Leissa has used the fundamental mode shapes of beams. However, in this study, a multiplication of free-free beam mode shape along $y$ and cosine mode shape along $x$ is used by assuming free edges and slip-shear support.

In Table 2, dimensionless damping ratios defined by Eq. (40) and the dimensionless damped frequency parameters defined by

$$
\lambda_{d m n}=\omega_{d m n} a^{2} \sqrt{\frac{\rho}{D}}
$$

for different Poisson's ratios are given.

It is clear that the springs and dampers at the four edges of the plate have an effect on the behaviour of the plate. In order to find an optimum value of the damping ratio in connection with the coefficients of spring and damper, one can look at how damping ratio being changed with respect to the change of spring and damper values. In Fig. 2, there are two curves showing that the damping ratio changes seperately depended on the spring and damper coefficients. The intersection point of these two curves is at the critical damping ratio which equals to one. In Fig. 3, the behaviour of damping ratio is plotted when the values of spring and damping are simultaneously changed. 


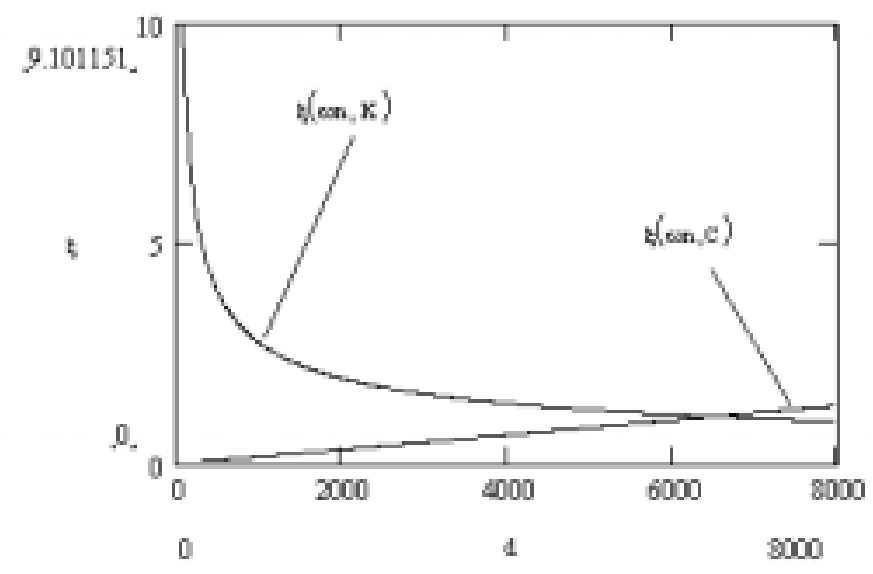

Fig. 2. The change of damping ratio versus to stiffness and damping for $\nu=0.225$.

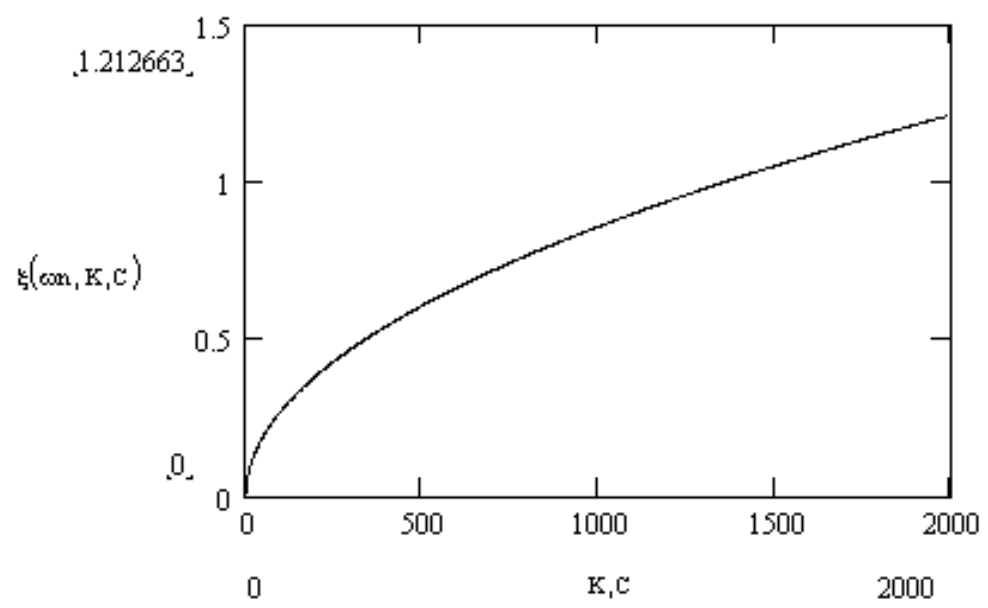

Fig. 3. The change of damping ratio as a function of stiffness and damping values for $\nu=0.225$.

In Table 3, the damping state for different Poisson's ratios is given with optimum values of the stiffness and viscous damping.

Different aspect ratios are also studied for Poisson's ratio $\nu=0.3$ and the results are tabulated in Table 4. Also, they are compared with the given values in the literature.

As expected, the dimensionless damping ratios and dimensionless damped frequency parameters change with the change of aspect ratios. Table 5 shows the results.

The optimum values of the stiffness and damping are related to plate behaviors which are determined by the material properties of the plate for free vibration analysis. In Table 6 , the damping state for aspect ratios $0.4,1.0$ and 2.5 with the density $\rho=2710 \mathrm{~kg} / \mathrm{m}^{3}$ and the modulus of elasticity $E=71 \times 10^{9} \mathrm{~N} / \mathrm{m}^{2}$ is given with the optimum values of the stiffness and viscous damping.

\section{Conclusions}

The classical theory is used for the equation of motion of a rectangular plate with the Kelvin type boundary conditions that include the stiffness and the viscous damping at four corners. An analytical solution procedure is 
Table 3

Damping state for different Poisson's ratios

\begin{tabular}{cccc}
\hline & \multicolumn{3}{c}{ Damping coefficient } \\
\hline$\nu=0.225$ & $<6030$ & 6030 & $>6030$ \\
Stiffness, & Under damped & Critically damped & Over damped \\
$\mathrm{K}=3.642118 \mathrm{E}+5$ & & & \\
$\nu=0.343$ & $<4720$ & 4720 & $>4720$ \\
Stiffness, & Under damped & Critically damped & Over damped \\
$\mathrm{K}=2.849 \mathrm{E}+5$ & & & \\
$\nu=0.360$ & $<4565$ & 4565 & $>4565$ \\
Stiffness, & Under damped & Critically damped & Over damped \\
$\mathrm{K}=2.793 \mathrm{E}+5 \nu=0.390$ & $<4295$ & 4295 & $>4295$ \\
Stiffness, & Under damped & Critically damped & Over damped \\
$\mathrm{K}=2.697 \mathrm{E}+5$ & & & \\
\hline
\end{tabular}

Table 4

Dimensionless frequency parameters $\lambda_{m n}$ for different aspect ratio $a / b$

\begin{tabular}{|c|c|c|c|c|c|c|}
\hline \multirow{2}{*}{$\begin{array}{c}\text { Mode } \mathrm{m} \\
\mathrm{m}\end{array}$} & \multicolumn{2}{|c|}{$a / b=0.4$} & \multicolumn{2}{|c|}{$a / b=1.0$} & \multicolumn{2}{|c|}{$a / b=2.5$} \\
\hline & & Leissa & & Leissa & & Leissa \\
\hline 1 & 3.7937 & 3.4629 & 13.687 & 13.489 & 24.668 & 21.643 \\
\hline 2 & 5.2294 & 5.2881 & 20.607 & 19.789 & 27.592 & 33.05 \\
\hline 3 & 10.911 & 9.622 & 25.422 & 24.432 & 54.266 & 60.137 \\
\hline 4 & 12.589 & 11.437 & 36.726 & 35.024 & 77.943 & 71.484 \\
\hline 5 & 19.712 & 18.793 & 36.726 & 35.024 & 109.17 & 117.45 \\
\hline 6 & 20.197 & 19.1 & 61.673 & 61.526 & 119.42 & 119.38 \\
\hline
\end{tabular}

Table 5

Dimensionless damping ratios and damped frequency parameters $\lambda_{d m n}$ for different aspect ratio $a / b$

\begin{tabular}{|c|c|c|c|c|c|c|}
\hline \multirow{2}{*}{$\begin{array}{c}\text { Mode } \\
\mathrm{m}\end{array}$} & \multicolumn{2}{|c|}{$a / b=0.4$} & \multicolumn{2}{|c|}{$a / b=1.0$} & \multicolumn{2}{|c|}{$a / b=2.5$} \\
\hline & $\xi$ & $\lambda_{d}$ & $\xi$ & $\lambda_{d}$ & $\xi$ & $\lambda_{d}$ \\
\hline 1 & 1.09 & 1.641 & 0.593 & 11.019 & 0.655 & 11.788 \\
\hline 2 & 0.271 & 5.493 & 0.266 & 19.863 & 0.265 & 16.825 \\
\hline 3 & 0.271 & 10.504 & 0.265 & 24.516 & 0.265 & 33.098 \\
\hline 4 & 0.266 & 12.136 & 0.264 & 35.423 & 0.264 & 47.547 \\
\hline 5 & 0.263 & 19.017 & 0.263 & 35.438 & 0.262 & 66.629 \\
\hline 6 & 0.259 & 19.506 & 0.259 & 59.567 & 0.259 & 72.951 \\
\hline
\end{tabular}

Table 6

Damping state for different aspect ratio $a / b$

\begin{tabular}{cccc}
\hline & \multicolumn{3}{c}{ Damping coefficient } \\
\hline$a / b=0.4$ & $<2562$ & 2562 & $>2562$ \\
Stiffness, & Under damped & Critically damped & Over damped \\
$\mathrm{K}=2.342488 \mathrm{E}+6$ & & & \\
$a / b=1$ & $<6405$ & 6405 & $>6405$ \\
Stiffness, & Under damped & Critically damped & Over damped \\
$\mathrm{K}=3.747981 \mathrm{E}+5 a / b=2.5$ & $<4295$ & 4295 & $>4295$ \\
Stiffness, & Under damped & Critically damped & Over damped \\
$\mathrm{K}=3.74681 \mathrm{E}+5$ & & & \\
\hline
\end{tabular}

proposed based on the modal analysis.

The completely free case of the continuous model of the plate is first studied. The expressions for the eigenfrequencies and eigenfunctions of the plate are obtained by utilizing the separation of variables. Following that, the stiffened and damped case is studied by using the Kelvin type boundary conditions by considering the spring and viscous damping as the external forces acting on the plate at the four corners. Then, the general solution to the equation of motion of this rectangular plate is obtained by following the modal analysis procedure. Finally, some numerical results are presented and compared with the values in the literature for accuracy. 


\section{Nomenclature}

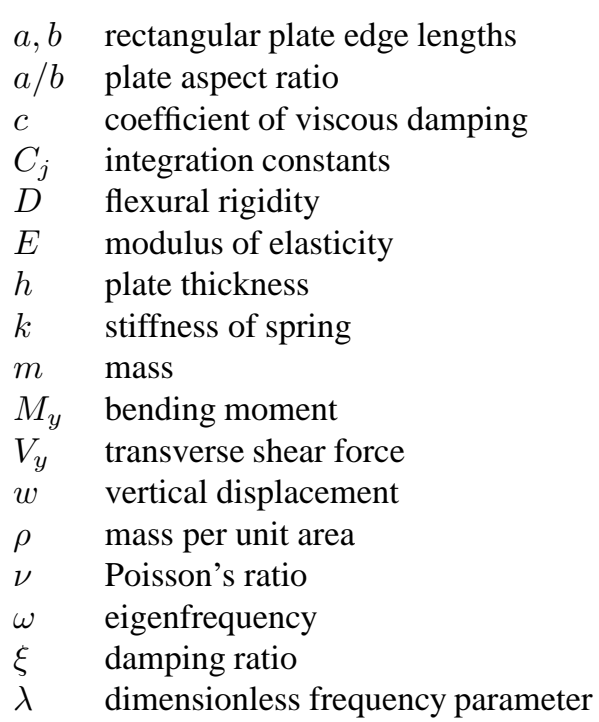

\section{References}

[1] D.J. Gorman, Free vibration analysis of the completely free rectangular plate by the method of superposition, Journal of Sound and Vibration 57(3) (1978), 437-447.

[2] D.J. Gorman, A general solution for the free vibration of rectangular plates with arbitrarily distributed and rotational elastic edge support, Journal of Sound and Vibration 174(4) (1994), 451-459.

[3] R.H. Gutierrez, P.A.A. Laura and R.O. Grossi, Vibrations of rectangular plates of bilinearly varying thickness and with general boundary conditions, Journal of Sound and Vibration 75(3) (1981), 323-328.

[4] D.J. Inman, Engineering Vibration, Prentice Hall, 2001.

[5] Y. Kato and T. Honma, The Rayleigh-Ritz solution to estimate vibration characteristics of building floors, Journal of Sound and Vibration 211(2) (1998), 195-206.

[6] P.A.A. Laura and R.O. Grossi, Transverse vibrations of rectangular plates with edges elastically restrained against translation and rotation, Journal of Sound and Vibration 75(1) (1981), 101-107.

[7] P.A.A. Laura and R.H. Gutierrez, Transverse vibrations of thin, elastic plates with concentrated masses and internal elastic supports, Journal of Sound and Vibration 75(1) (1981), 135-143.

[8] A.W. Leissa, Vibration of Plates, Ohio State University, Columbus, Ohio.

[9] A.W. Leissa, The free vibration of rectangular plates, Journal of Sound and Vibration 31(3) (1973), 257-293.

[10] A.W. Leissa, P.A.A. Laura and R.H. Gutierrez, Vibrations of rectangular plates with nonuniform elastic edge supports, Journal of Applied Mechanics 47 (1980), 891-895.

[11] C. Rajalingham, R.B. Bhat and G.D. Xistris, Vibration of rectangular plates by reduction of the plate partial differential equation into simultaneous ordinary differential equations, Journal of Sound and Vibration 203(1) (1997), 169-180.

[12] R.K. Singhal and D.J. Gorman, Free vibration of partially clamped rectangular plates with and without rigid point supports, Journal of Sound and Vibration 203(2) (1997), 181-192.

[13] Y.B. Zhao and G.W. Wei, DSC analysis of rectangular plates with non-uniform boundary conditions, Journal of Sound and Vibration 255(2) (2002), 203-228. 

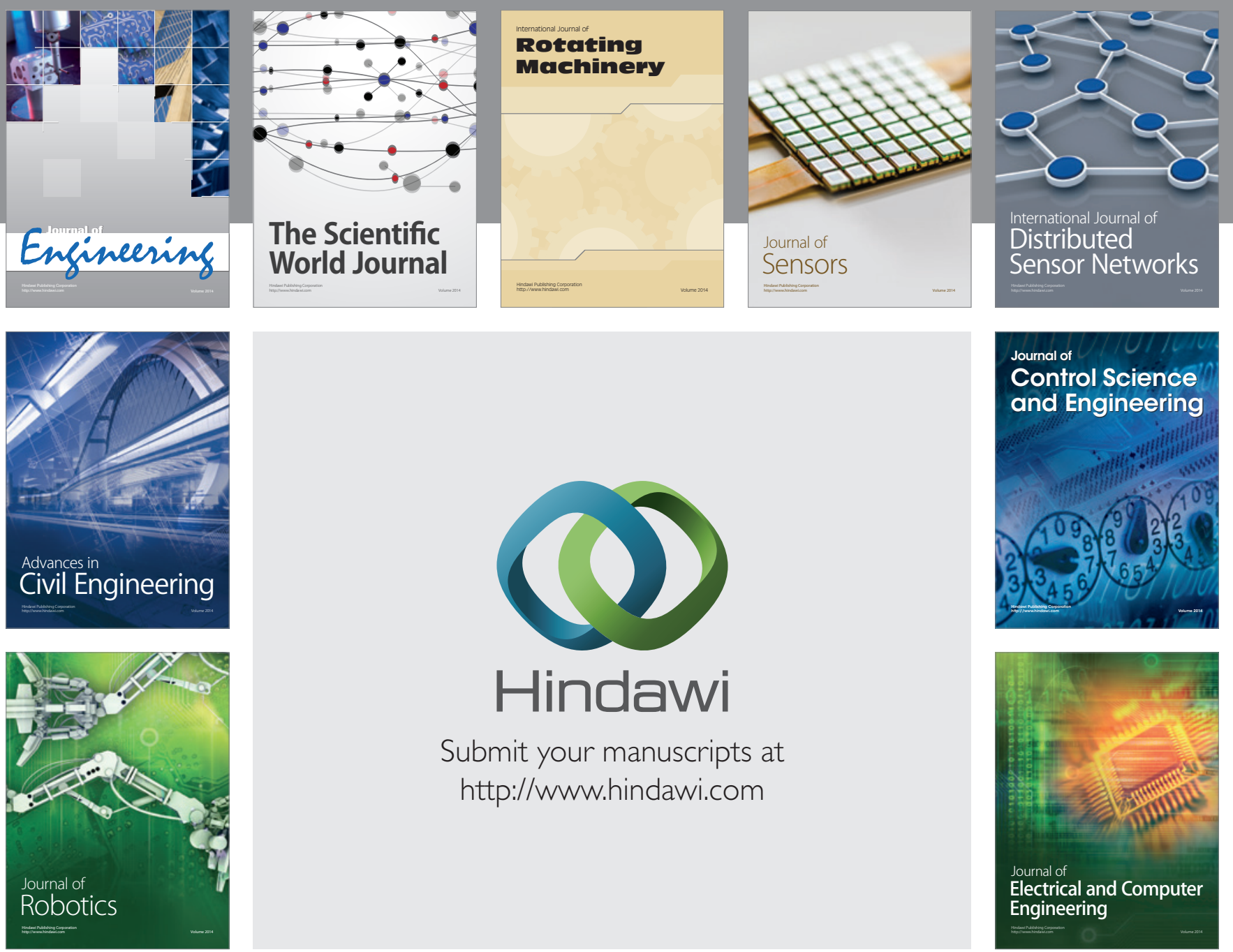

Submit your manuscripts at

http://www.hindawi.com
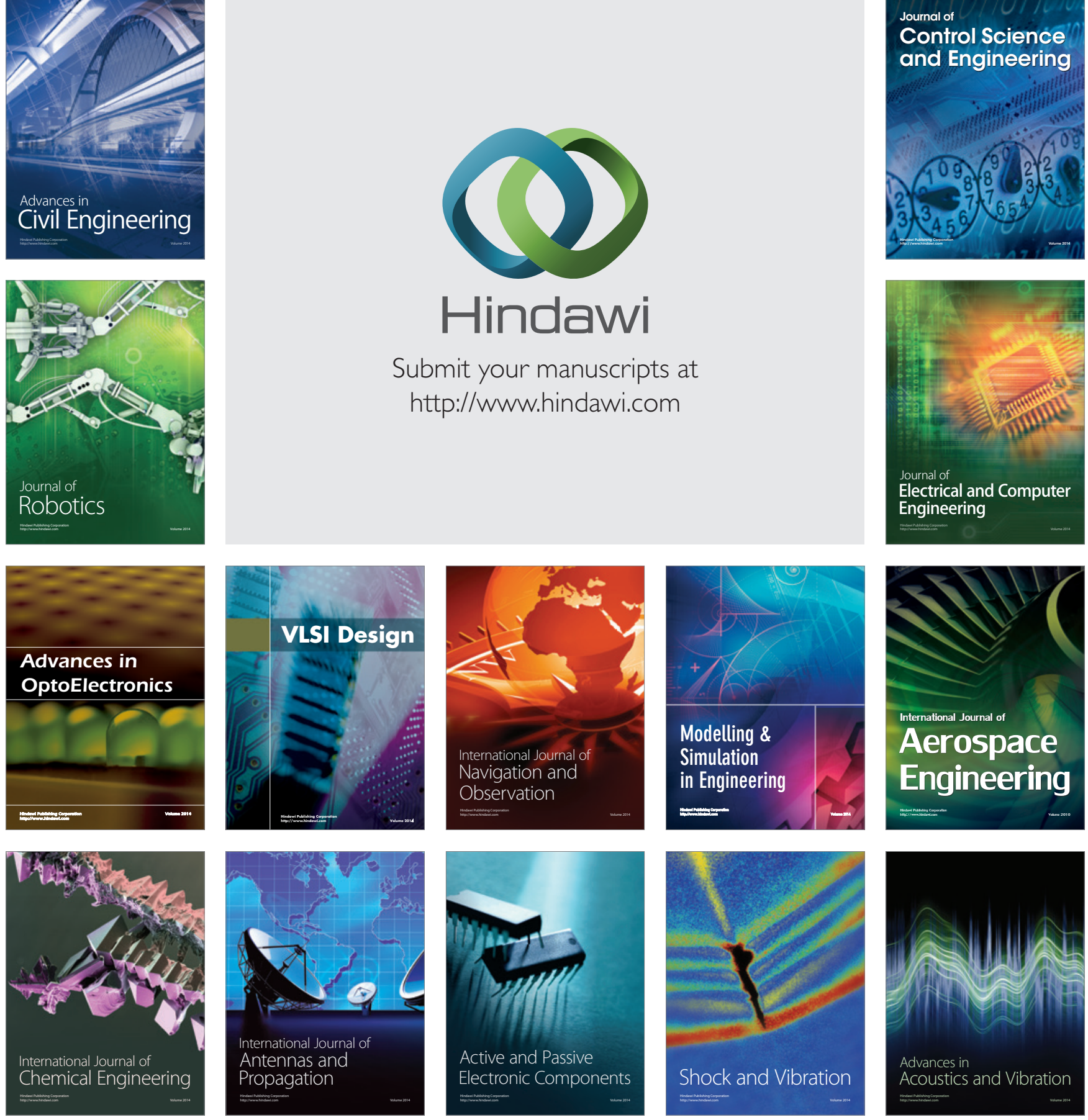